\title{
La science-fiction du lisible au visible \\ L'exemple du film «La stratégie Ender » de Gavin HOOD
}

\author{
Tarnime Mohamed Réda Taher ${ }^{1}$ \\ Tarnime_reda@alexu.edu.eg
}

\section{Résumé:}

L'objectif de cet article est de démontrer que des liens solides associent l'art littéraire à l'art filmique. Dans le cadre d'une approche comparative, nous effectuons un mouvement alternatif entre le verbal et le visuel. Après avoir étudié les spécificités de chacune des deux structures artistiques, nous avons jugé important de fonder notre étude sur l'exemple de l'adaptation du roman La stratégie Ender afin d'étudier les techniques de mise en scène les plus significatives se rapportant au domaine science-fictionnel. D'une part, le septième art a constamment puisé dans la littérature pour se nourrir. D'autre part, si l'œuvre possède une densité notable, elle s'enrichit surtout à être réinterprétée et transposée à l'écran. Nous avons donc constaté que le cinéma impose une profusion de détails particuliers par le truchement de maints procédés de réalisation: casting, décor, lumière, cadrage, échelle des plans, angle de prise de vue, procédés multidimensionnels, costumes, musique, rythme. L'analyse de ces procédés de mise en scène nous a permis d'éclairer la richesse latente de la manipulation cinématographique de l'œuvre littéraire.

Mots-clés: Cinéma de science-fiction; adaptation cinématographique; littérature et cinéma.

1 Maître de conférences, Département de langue française, Faculté de Pédagogie, Université d'Alexandrie.

(La science-fiction du lisible au visible ...) Dr. Tarnime Mohamed Réda 
«La mise en scène n'est plus un moyen d'illustrer ou de présenter une scène, mais une véritable écriture. L'auteur écrit avec la caméra comme un écrivain avec son stylo ${ }^{(1)}$.

Le septième art a depuis toujours manifesté un engouement particulier envers la littérature et les cinéastes ne cessent d'y puiser leurs principales sources d'inspiration grâce à la richesse infinie de son patrimoine. D'emblée, le cinéma science-fictionnel chante les topoï du progrès technologique et offre une large gamme de représentations fictives reposant sur la modernisation que nous vivons à l'âge du numérique actuel. La science-fiction est incontestablement l'unique genre littéraire apte à se réapproprier la numérisation qui envahit nos sociétés et à l'extrapoler vers les contrées imaginaires. Fondée spécifiquement sur ce potentiel séduisant, l'adaptation cinématographique pourrait être perçue comme la mise en œuvre visuelle de ces motifs que ce soit dans la manipulation figurative, temporelle ou spatiale. D'ailleurs, les relations entre l'art filmique et l'art littéraire sont nombreuses. Toutefois, lire un livre et visionner un film sont deux actes complètement différents. En quoi consiste chaque entreprise? Quelles en sont les composantes primordiales? Quelle est l'étape fondamentale de la transposition de l'œuvre à l'écran? Quels sont les procédés techniques de la mise en scène? Quelles sont les particularités dans le cas d'un roman de fiction? Telles sont les questions qui orienterons notre recherche.

(La science-fiction du lisible au visible ...) Dr. Tarnime Mohamed Réda 
Dans le présent article, nous proposons une réflexion sur la transposition de l'œuvre littéraire au cinéma et nous examinerons cette entreprise particulièrement dans le cas d'un roman de science-fiction. Nous fonderons essentiellement notre étude sur l'exemple de l'adaptation de La stratégie Ender ${ }^{(2)}$ de l'écrivain américain Orson Scott Card réalisée en 2013. Dans le cadre d'une approche comparative, nous effectuerons des parallèles entre le livre et le film afin d'examiner et de discerner les incarnations figuratives des tropes fictifs. Cet angle de vue distinct nous permettra d'effectuer « un déplacement créateur [...] d'un univers dans un autre » ${ }^{(3)}$. Il est indéniable que le cinéma possède une panoplie de techniques: lumières, sons, mouvements, cadrage, montage, gestes, dialogues, etc. Nous tenterons d'étudier ces diverses stratégies de réalisation et de transposition des cadres et des actions scrutés par l'écrivain dans l'espace romanesque, concrétisés par le cinéaste dans le film afin d'évaluer l'apport du réalisateur.

\section{Le verbal VS le visuel:}

«Indépendamment l'un de l'autre, le roman et le film de fiction peuvent être conçus comme des systèmes, [...] comme des ensembles autonomes constitués d'éléments distinctifs subordonnés à une constante interaction $\gg{ }^{(4)}$. 
Le littéraire et le filmique se rejoignent en quelques aspects mais se distinguent au niveau de plusieurs autres composantes. Par définition, le cinéma est un art mettant en œuvre des procédés spéciaux dans le but de projeter photographiquement des vues animées; c'est également l'art de composer et de réaliser des films. Cette entreprise se fonde sur une représentation visuelle concrète. Dans ce cas, le spectateur est placé dans une position de passivité comme à l'état d'un rêve apparent où l'image serait inchangeable par le truchement d'une communication à sens unique. Or, la lecture est une activité personnelle basée sur la représentation mentale que chaque individu se fait du récit. Elle offre un large éventail de possibilités de fantaisie sans limite. Dans le cas de la fiction scientifique, puisqu'il s'agit d'imaginer des créatures extraterrestres ou des univers interstellaires, les images mentales que se forgent les lecteurs semblent être de plus en plus fantasmagoriques. Lire de la science-fiction implique donc une participation plus active de la part du lectorat au niveau de l'imagination.

De plus, les deux systèmes sémiotiques sont carrément opposés et emploient des matériaux signifiants différents. Comme l'auteur juxtapose les mots et fait appel aux phrases, le cinéaste recourt aux scènes et aux séquences. Le potentiel narratif et la logique des signes sont clairement distincts. Le rôle du réalisateur consiste à recomposer l'œuvre littéraire dans un mode 
d'expression nouveau dans lequel le cadre, le bruitage, la musique et les mouvements seraient les principaux fondements. Dans la production filmique, la bande-image assume à elle seule la narration, comme si l'histoire se contait seule de manière autonome et immédiate sans aucun narrateur identifiable. Si ce dernier jouit d'un statut privilégié dans le récit, son rôle pourrait aisément être négligé dans la transposition visuelle au profit d'une instance narrative spécialisée au langage cinématographique.

Il importe également de souligner le caractère linéaire du signe linguistique car chaque mot opère comme "relais de signification » ${ }^{(5)}$ permettant d'exprimer de multiples réalités concrètes ou abstraites et contribue à la signification globale du texte. Néanmoins, dans le cas du film, l'image est polysémique et présente aux spectateurs des signifiants multiples et synchrones qui interagissent simultanément. Le cinéma de fiction notamment repose sur des effets spéciaux capables d'allécher son public qui en manifeste un intérêt particulier.

De surcroît, le facteur du temps constitue à son tour un élément de dissemblance. D'une part la lecture nécessite une certaine durée, laquelle pourrait s'étaler sur plusieurs jours ou parfois des semaines. Le lecteur jouit d'une certaine liberté qui lui permet de parcourir quelques passages, de relire d'autres, de revenir en arrière ou de sauter des digressions. De l'autre, le film quant à lui est voué à être condensé en deux ou trois heures 
maximum. Il impose au spectateur un rythme et un enchaînement dans la narration. Cette disproportion implique des remaniements imposés par le processus de transposition filmique sur le récit original tel que l'amalgame ou la suppression de quelques séquences narratives puisque «transposer à l'écran en deux heures de projection une cuvre de cinq cents pages, c'est inévitablement réduire, résumer, dénaturer ${ }^{(6)}$.

Sur un autre plan, le romancier est l'unique créateur du récit. L'acte d'écriture est une activité individuelle qui se déclenche devant la page blanche. L'auteur se met à dessiner sur le papier des personnages et des péripéties par le truchement de la langue. En revanche, la production cinématographique s'élabore à de multiples niveaux: scénario, appareillage, techniciens, caméramans, et acteurs. "Le film est une entreprise collective associant des dizaines sinon des centaines de professionnels ${ }^{(7)}$. Chaque spécialiste emploie son matériau et ses outils afin de marquer sa propre empreinte dans la réalisation de l'image et contribue à la dimension spectaculaire du film.

Il faut donc admettre qu'il n'existe aucune équivalence entre les énoncés linguistiques, unité de base du langage littéraire et l'image/son substance de la production cinématographique. « L'adaptation revendique un dialogue ente le texte littéraire et le texte filmique dans lequel s'établit le sens direct des ouvres en interaction ${ }^{\left({ }^{(8)}\right.}$. La genèse de chaque œuvre étant autonome, la 
création d'un film à partir d'un roman équivaudrait donc à une manipulation afin d'aboutir à une réincarnation interprétative du roman.

\section{Les composantes du récit: personnages et télescopage} spatio-temporel:

«Ainsi, le système romanesque et le système filmique s'érigent-ils en partie sur la base du réseau de relations entre les personnages et les situations qui évoluent dans le temps et dans l'espace de la diégèse représentée ${ }^{(9)}$.

Nous arrivons à la composante principale des deux types de récit à savoir les personnages. Ils sont le noyau autour duquel gravite toute l'histoire puisque c'est à travers eux que les évènements se passent. Soit qu'ils provoquent les actions soit qu'ils les subissent, ils demeurent des héros auxquels le lecteur pourrait s'identifier en s'appropriant le récit et en les incarnant selon son imaginaire personnel. Cependant, le cinéma freine l'imagination des lecteurs. Les protagonistes sont imposés à travers le choix du casting. Ils sont des êtres en chair et en os, joués par des acteurs qui sont forcément différents des images générées pendant la première lecture. Cette disparité s'amplifie lorsqu'il s'agit d'un récit de fiction car en ce cas les personnages pourraient être réalisés complètement à travers des modalités techniques comme la dimension 3D.

(La science-fiction du lisible au visible ...) Dr. Tarnime Mohamed Réda 
Comme nous l'avons déjà mentionné, dans les récits visuels, les séquences narrées sont remplacées par les mêmes actions rendues visibles. Le rôle du narrateur qui était primordial dans les récits scripturaux s'estompe au profit des effets audiovisuels. C'est ce caractère polysémique qui assure au septième art son aspect attrayant. Conjointement, si le cinéma est voué à présenter le réel dans son extériorité par le truchement de la gestuelle, des expressions du visage des protagonistes, le roman par contre n'emploie que les mots pour exprimer l'intériorité des personnages, leurs sentiments ou leurs désirs. Ainsi, dans l'adaptation, sont écartés plusieurs développements textuels accessoires concernant les sensations des héros, leurs imaginations ou leurs angoisses.

De plus, au cinéma, le facteur du temps est fort important car la rédaction d'un roman suit l'ordre chronologique. Toutefois, le cinéma est un art de la discontinuité. À la narration linéaire de l'écrit se substituent des techniques de mise en scène comme le flash-back ou le fondu enchainé qui permettent des variations dans le rythme et assurent une certaine malléabilité sur l'axe temporel.

En ce qui concerne la dimension spatiale qui est pour une grande part présentée dans le récit par le biais de passages descriptifs, elle est également éliminée et remplacée par les studios qui permettent de subordonner l'élément visuel au verbal. 
L'univers qui abrite les personnages fictifs est à son tour profondément singulier. Les contrées interstellaires sont transposées dans des studios à décors accrocheurs afin d'exploiter les fonds de ce cosmos et de le transcrire. Les espaces tridimensionnels (3D) sont également très significatifs à ce sujet.

C'est dans cette perspective que le cinéaste pourrait être considéré comme étant un homologue de l'écrivain dans la mesure où le processus d'adaptation nécessite la recomposition de l'œuvre littéraire à l'aide de divers moyens d'expression spécialisés.

\section{3- La scénarisation:}

«Le scénariste doit [...] élargir le champ romanesque, voire le modifier mais sans pour autant le dénaturer» ${ }^{(10)}$.

L'interaction entre les deux types de récit franchit une phase intermédiaire qui permet de transcoder les constituants du roman. L'œuvre littéraire passe tout d'abord par la lecture ensuite l'interprétation pour aboutir à la création du scénario. Cette dernière est la première passerelle à accomplir afin de garantir une homogénéité des éléments cinématographiques. Ainsi, indépendamment des qualités artistiques de l'adaptation, la scénarisation développe un discours de réécriture en marge du texte, adossé au travail du romancier mais en même temps asymétrique, indépendant et original. À travers le script, le récit

(La science-fiction du lisible au visible ...) Dr. Tarnime Mohamed Réda 
littéraire est remanié et morcelé avant de se munir de l'empreinte filmique.

La première étape dans la création du scénario consiste en un découpage narratif délimitant les différentes scènes de manière discontinue tout en assurant la cohérence du tissu fictif. Ce stade vise à transposer l'esprit général de l'œuvre et à faire revivre le texte littéraire en le divisant en une série de séquences et en retenant les épisodes essentiels de la trame narrative. L'adaptateur jouit d'une autorité complète, il pourrait donc resserrer des actions, simplifier les intrigues, réduire le nombre de personnages, dramatiser les scènes, enrichir les dialogues, accentuer certains caractères spéciaux, amplifier des effets de suspense; bref, reconstruire le récit différemment et manifester sa marque esthétique en actualisant la «logique signifiante éparpillée dans le roman ${ }^{(11)}$.

C'est pour cette raison que le travail scénaristique a toujours été suspecté de réduire le potentiel symbolique de l'œuvre littéraire. Or, considéré comme étant «un document à usage interne » ${ }^{(12)}$, méconnu par le large public, le script constitue l'essentiel intermédiaire entre le texte du romancier et la création du cinéaste. Ceci se réalise en entreprenant une quête des équivalents du langage verbal par le biais des images, d'une redistribution des significations latentes de cette production romanesque originale dans un processus d'effacement du lisible 
au profit du visible. Raconter au cinéma serait donc synonyme de « donner à voir ».

Parmi les spécificités du scénario, nous retenons cinq composantes élémentaires: les personnages, l'objectif, le conflit, le dialogue et la structure qui soutiennent la progression dramatique de l'intrigue et se donnent à voir au cours de trois actes. En premier lieu, dans l'exposition les spectateurs font la connaissance du protagoniste et de l'objet de sa quête, des objectifs qui le motivent et des obstacles qui l'entravent. En second lieu, le développement présente une partie intrinsèque du scénario qui expose la tension dramatique et les péripéties allant crescendo. Ces épisodes introduisent le climax et visent à provoquer une participation émotionnelle accrue chez le spectateur tout en le séduisant. En dernier lieu, dans le dénouement s'exhibe la résolution du conflit et ses conséquences où le personnage pourrait atteindre les objectifs ou se révéler voué à l'échec. Cet épilogue véhicule la morale du film selon la vision du cinéaste indépendamment de celle du romancier.

Dans les volets qui vont suivre, nous tenterons de souligner les rapports singuliers qui s'établissent entre le corpus choisi $L a$ stratégie Ender et son adaptation tout en prenant en compte que le réalisateur du film Gavin Hood est lui-même le scénariste. Notre objectif sera de mettre l'accent sur le fait que le septième art fonde sa dimension prodigieuse sur des procédés fort particuliers 
susceptibles d'impressionner, de convaincre et d'enthousiasmer son public. Phénomène lucratif fascinant, l'adaptation est fondée sur «une stratégie marketing » ${ }^{(13)}$ qui fait revivre le roman et lui confère une nouvelle allure.

\section{Synopsis:}

« Le système filmique possède [...] la propriété de placer le spectateur dans une situation où il doit absorber une masse importante d'information dans un laps de temps restreint ${ }^{(14)}$.

Effectivement, le littéraire et le cinématographique s'opposent au niveau des objectifs. Il est indéniable que le cinéma de science-fiction repose essentiellement sur des effets spectaculaires. Voilà pourquoi, nous nous sommes orientés vers la transposition du roman La stratégie Ender. En fait, le choix de cette réalisation présente nombreux intérêts. Adaptation d'un bestseller, ce film relate l'histoire d'un enfant doté d'une intelligence hors du commun chargé de sauver la planète Terre contre l'attaque d'une race insectoïde nommé les Doryphores. Dans la première offensive, les humains ont gagné grâce à l'héroïsme de Mazer Rackham qui commandait la Flotte Internationale. Depuis, le colonel Graff, en tête des forces militaires terriennes, entreprend la tâche d'entraîner les enfants les plus prometteurs afin de s'apprêter au combat.

(La science-fiction du lisible au visible ...) Dr. Tarnime Mohamed Réda 
Observé dès son plus jeune âge, Andrew Wiggin, surnommé Ender, est envoyé dans un vaisseau spatial et appelé à intégrer l'école de guerre où les jeunes suivent un entraînement excessif pour être prêts à affronter l'ennemi dans la bataille intersidérale. Entre opéra de l'espace et cadres intergalactiques, le récit scrute l'itinéraire de la construction d'une flotte d'enfants guerriers, solidement formés dans un contexte où toute minute compte. Il faut éradiquer la menace, atteindre l'objectif ultime et gagner à tout prix. Détruire l'ennemi devient ainsi l'unique lueur d'espoir pour sauvegarder la planète et garantir la survie de l'humanité. L'anéantissement des Doryphores semble être inéluctable.

Aussi, suivons-nous de très près l'évolution de ces élèvesofficiers. Au cours du périple, nous assistons à une série de simulations de guerre afin de leur assurer un entraînement solide. Le film met surtout l'accent sur les relations enfants/adultes spécialement dans un contexte animé par les angoisses et les questionnements qui coïncident avec le processus d'encadrement militaire. Toutefois, l'épilogue présente une fin ouverte et appelle à une suite. La communication avec les Doryphores pourrait-elle amener à la reconstruction?

\section{Procédés de réalisation:}

« Tous [1]es constituants spécifiquement filmiques ne se trouvent pas dans le texte littéraire:

(La science-fiction du lisible au visible ...) Dr. Tarnime Mohamed Réda 
ils constituent une richesse propre au cinéma, susceptible d'apporter au livre initial une résonance nouvelle, que ce soit sur le plan des significations, sur celui de la construction narrative, et surtout en matière de pouvoir émotif» ${ }^{(15)}$.

L'industrie cinématographique doit éblouir les yeux tout en mobilisant l'intellect. De ce fait, elle nécessite un investissement financier fort coûteux. C'est la raison pour laquelle les grands cinéastes se sont toujours inspirés de romans accrocheurs en leur assurant une richesse supplémentaire et une allure impressionnante afin de mener à bien des films marquants et d'obtenir en même temps de grands succès commerciaux. Ainsi, cet art $\mathrm{du}$ spectacle constitue une «véritable expérience sensorimotrice » ${ }^{(16)}$ mettant en œuvre divers procédés techniques. Le cinéma impose sa narrativité exceptionnelle fondée sur le langage des mouvements de la caméra, du choix des décors, des échelles de plans et du cadrage, des angles de prise de vue et du rythme que nous soulignerons au cours des volets suivants. Sans vouloir chercher dans le film l'œuvre adaptée, nous nous concentrerons sur l'apport du cinéaste. Or, l'étude exhaustive des diverses techniques de mise en scène ne pouvant s'effectuer dans le cadre limité de cette analyse, nous nous contenterons de signaler les exemples qui nous paraissent les plus pertinents afin d'illustrer notre propos.

(La science-fiction du lisible au visible ...) Dr. Tarnime Mohamed Réda 


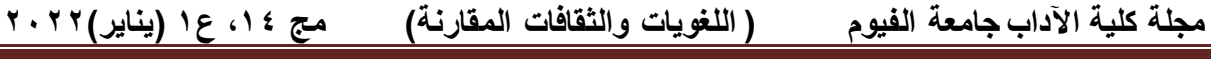

\section{A. Le casting:}

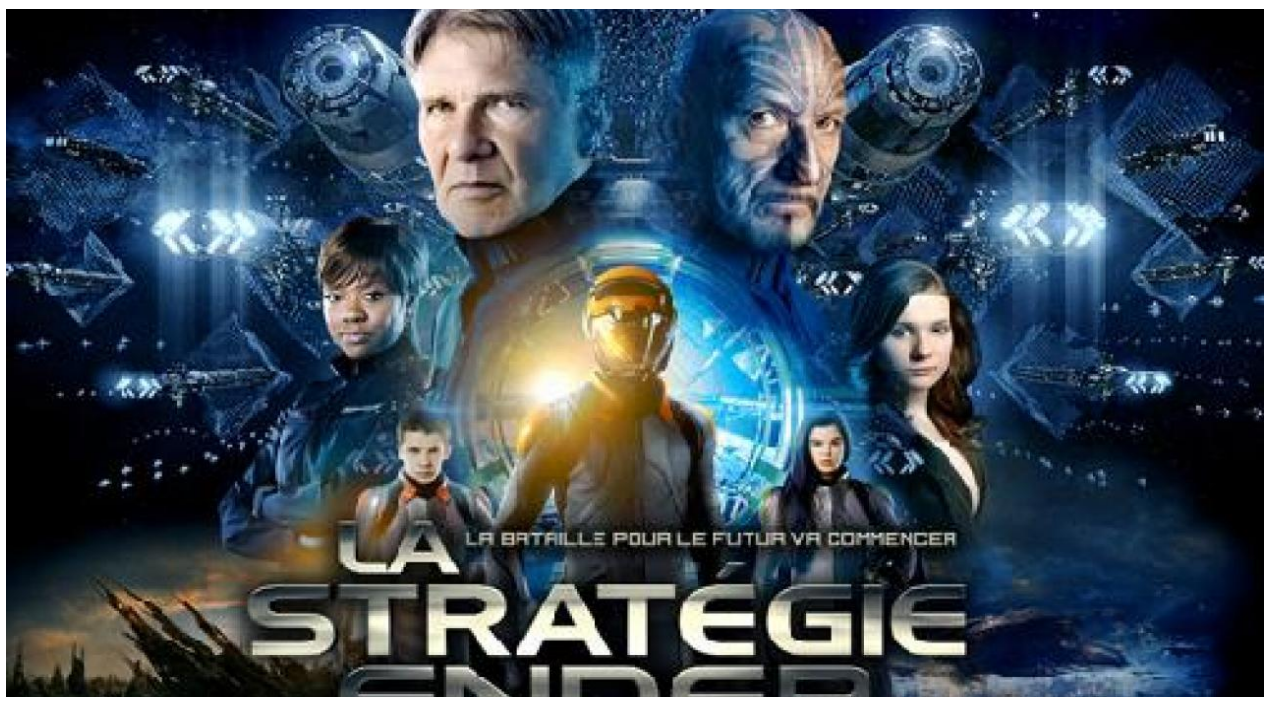

(17)

«Il est temps pour le cinéaste de sélectionner les comédiens qui incarneront les différents personnages de l'histoire ${ }^{(18)}$. Le premier point à analyser est celui des acteurs qui prêtent aux caractères leurs traits, leur silhouette et leur gestuelle. Bien qu'il ait témoigné maints changements au niveau des acteurs, ce film présente un casting solide.

Si les noms des jeunes américains Jake Lloyd ou Nathan Gamble étaient suggérés pour incarner le personnage d'Ender, c'est finalement le britannique Asa Butterfield qui s'empare du choix du réalisateur Gavin Hood. En effet, Ender est un personnage à caractère complexe qui se métamorphose d'un gamin coléreux, rageur et agressif en un chef de guerre capable de diriger les situations les plus difficiles. Butterfield, âgé de 16 ans,

(La science-fiction du lisible au visible ...) Dr. Tarnime Mohamed Réda 
qui a déjà été découvert dans le film Hugo Cabret de Martin Scorsese, représente un choix intelligent, impressionnant et surtout convaincant. En effet, la raison pour laquelle le réalisateur a renoncé à projeter à l'écran un acteur de six ans comme le présente le roman est surtout que ce dernier abonde en passages extrêmement violents à tous les niveaux: la violence physique à travers les agressions que le jeune enfant subit de son frère Peter et la violence morale avec les intimidations continuelles de ses camarades. Le film expose notamment la dimension intime de la vie de l'enfant prodige et aborde les implications et les séquelles des destructions psychologiques qu'il affronte tout en soulignant sa tactique de pensée, sa stratégie guerrière et ses réflexes dans les simulations. Attribuer à un enfant le rôle du héros aurait été une entreprise risquée. Voilà pourquoi la nomination de ce jeune acteur pour substituer le personnage principal du roman s'est révélée plus appropriée. Le regard et les expressions faciales de Butterfield sont parfaitement suffisants afin d'illustrer la détermination et l'obstination d'un tacticien de guerre. 


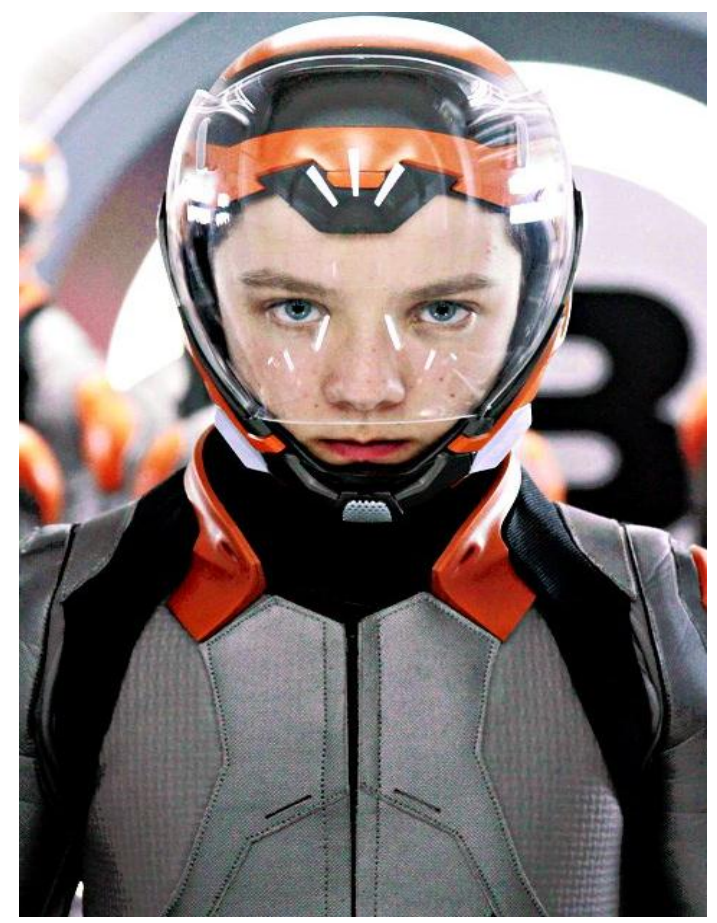

Le colonel Hyrum Graff est interprété par Harrison Ford, éminente figure dans le cinéma d'action. C'est lui qui repère l'élève prometteur et entreprend sa manipulation en lui donnant confiance en ses capacités. Le général Mazer Rackham, vainqueur de l'attaque initiale, est joué par Ben Kingsley. Au physique fortement connoté et exagérément tatoué, il est doté d'un regard perçant et des traits d'une excessive agressivité. C'est lui qui assume la tâche de la formation du futur héros.

(La science-fiction du lisible au visible ...) Dr. Tarnime Mohamed Réda 


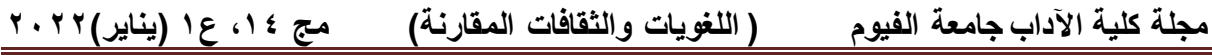
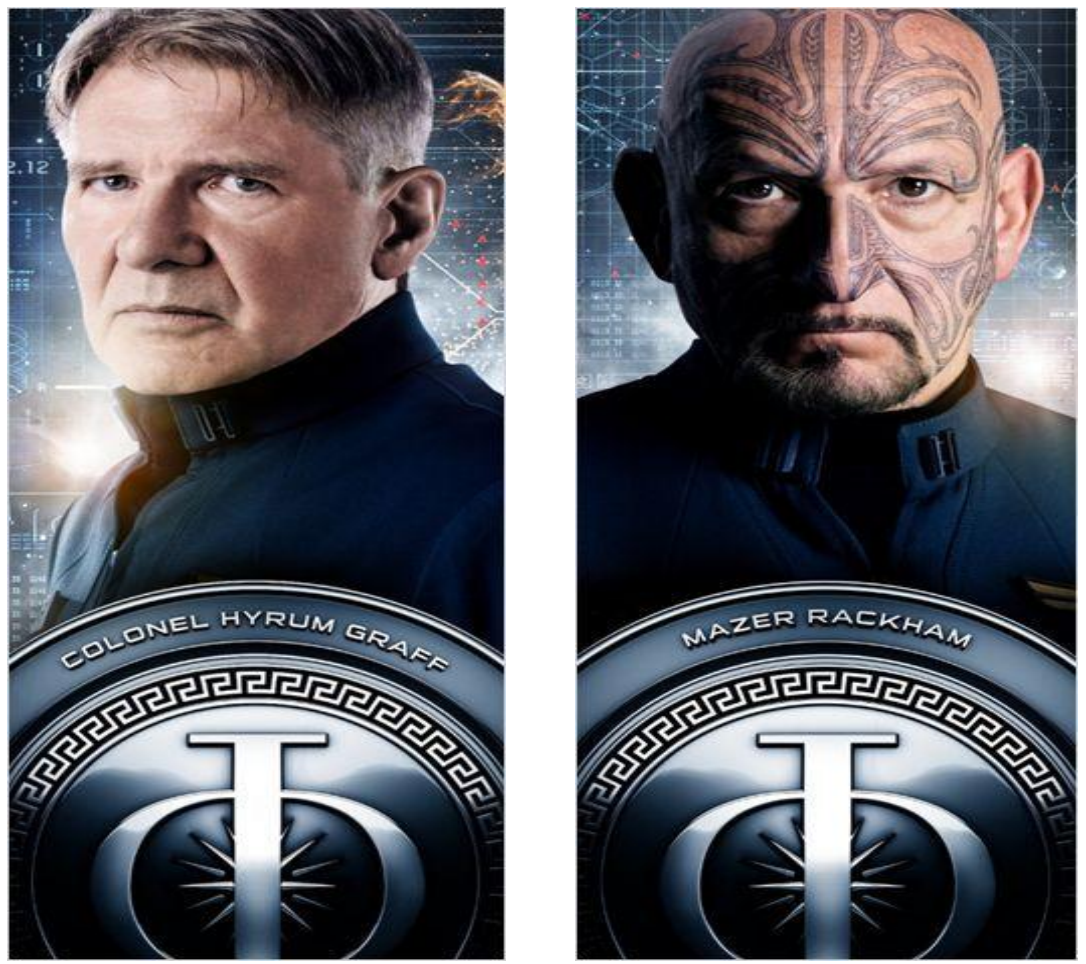

(19)

Quant aux figures féminines, l'actrice Abigail Breslin est sollicitée pour jouer le rôle de Valentine, la sœur aînée d'Ender et Hailee Steinfeld pour interpréter Petra Arkanian, la collègue qui l'épaule à l'école de guerre et l'encourage dans l'entraînement. L'une et l'autre assument une fonction de soutien pour Ender Wiggin; la première sur le plan affectif et la seconde sur le plan militaire. Finalement, l'actrice américaine Viola Davis incarne le commandant Gwen Anderson, adjointe du général Graff.

(La science-fiction du lisible au visible ...) Dr. Tarnime Mohamed Réda 


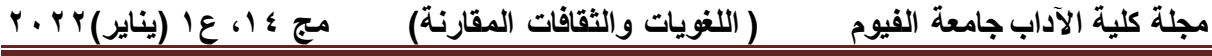

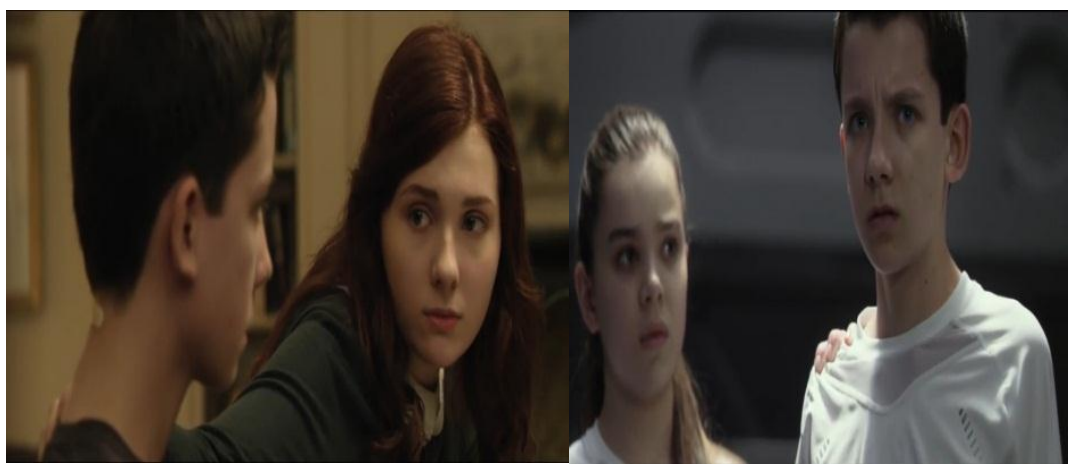

Ender avec Valentine Wiggin Ender avec Petra Arkanian

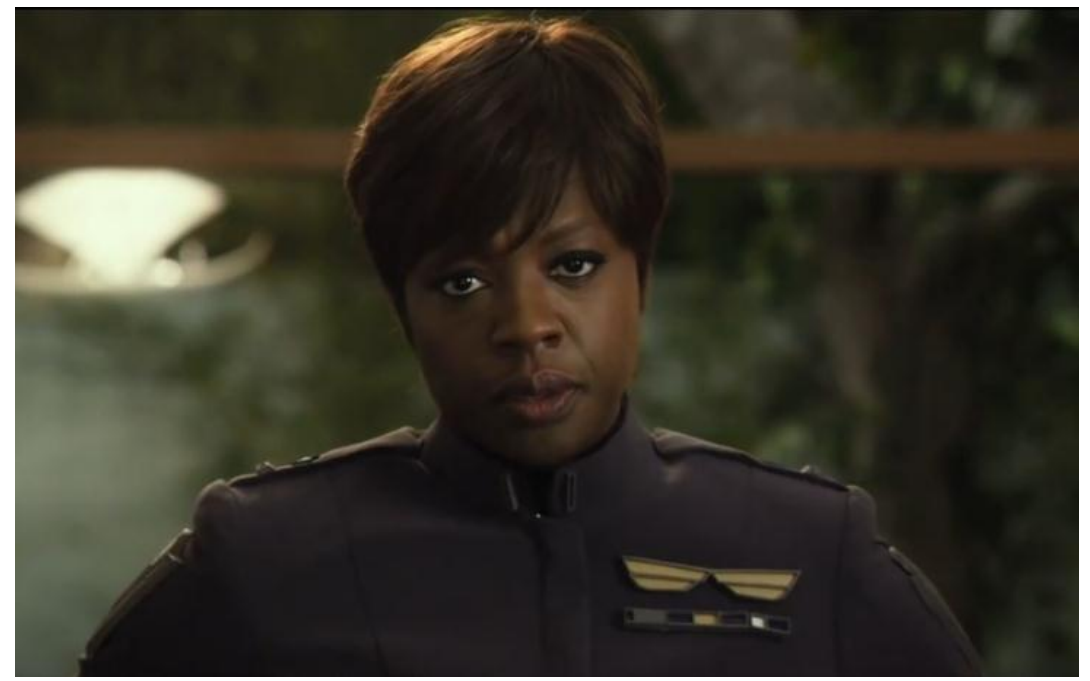

Major Gwen Anderson

Ainsi s'effectue la distribution des rôles des principaux protagonistes. En somme, «la sélection du comédien à partir de tel personnage de fiction est en soi tout un art ${ }^{(20)}$. Le casting $\mathrm{du}$ film est crédible et alléchant, le choix des figures féminines est surtout judicieux.

Il reste à noter une composante principale du scénario et indissociable des personnages: celle des dialogues filmiques qui

(La science-fiction du lisible au visible ...) Dr. Tarnime Mohamed Réda 
assument maintes fonctions dans la progression des actes. Ils transmettent les informations narratives, laissent entrevoir quelques traits de caractères moraux, véhiculent des détails supplémentaires et présentent aux spectateurs diverses indications relatives aux événements antérieurs indispensables à la compréhension de la situation. Citons à titre d'exemple la scène où la famille Wiggin est à table après le retirement du moniteur qui était chargé de surveiller les réflexes du héros:

- Ender: «Je suis un troisième, je n'aurais jamais dû venir au monde ».

- La mère: «Non, on t'a désiré Ender. On a juste dû obtenir l'autorisation ».

Dans cette séquence, le jeune enfant déçu nous fait part de la tristesse due à sa situation comme étant un troisième enfant dans une société surpeuplée qui applique un strict contrôle sur le taux de naissance et qui interdit aux parents d'en avoir plus que deux sans la permission de l'État. Cette scène dévoile le motif du choix du nom du héros puisque le terme « ender » en anglais signifie le dernier. Andrew est le benjamin de la famille et le dernier combattant qui sauvera l'humanité. C'est dans cette mesure que les acteurs se comportent et emploient un langage qui s'enrichit par échelons au moyen des actions et des paroles qu'ils s'échangent. Ces dialogues réalistes et crédibles soutiennent la progression de l'intrigue et servent à faire avancer l'histoire. 
B. Le champ visuel:

Il est indéniable que le récit filmique présente une perception accentuée par rapport au livre. Les pauses descriptives dans le roman sont transmises au cinéma par le biais d'une panoplie d'images qui désignent, racontent et décrivent simultanément. De ce fait, pour que l'adaptation puisse être rentable, le metteur en scène doit habilement manipuler le champ visuel, animé par un rayonnement nouveau et une créativité au niveau des représentations imagées.

1. Les décors:

«Inspirés par la beauté et la splendeur du cosmos et par tout ce qu'implique la perspective de voir l'humanité quitter sa planète ancestrale, les artistes d'aujourd'hui créent de nouvelles formes d'expression adaptées à l'expansion humaine dans cet environnement nouveau » ${ }^{(21)}$. Dans La stratégie Ender, l'espace offert au spectateur est dirigé par Sean Haworth, directeur artistique américain. Les décors du film, dont la majorité constitue des salles de jeu où se déroulent les entraînements, sont réalisés par A. Todd Holland. En général, les stations spatiales opèrent en tant que bases militaires. Cette ambiance de réalité virtuelle constitue l'épicentre de la fiction scientifique et prédomine dans les systèmes interactifs de simulation de guerres et de combats par le biais d'images tridimentionnelles.

(La science-fiction du lisible au visible ...) Dr. Tarnime Mohamed Réda 


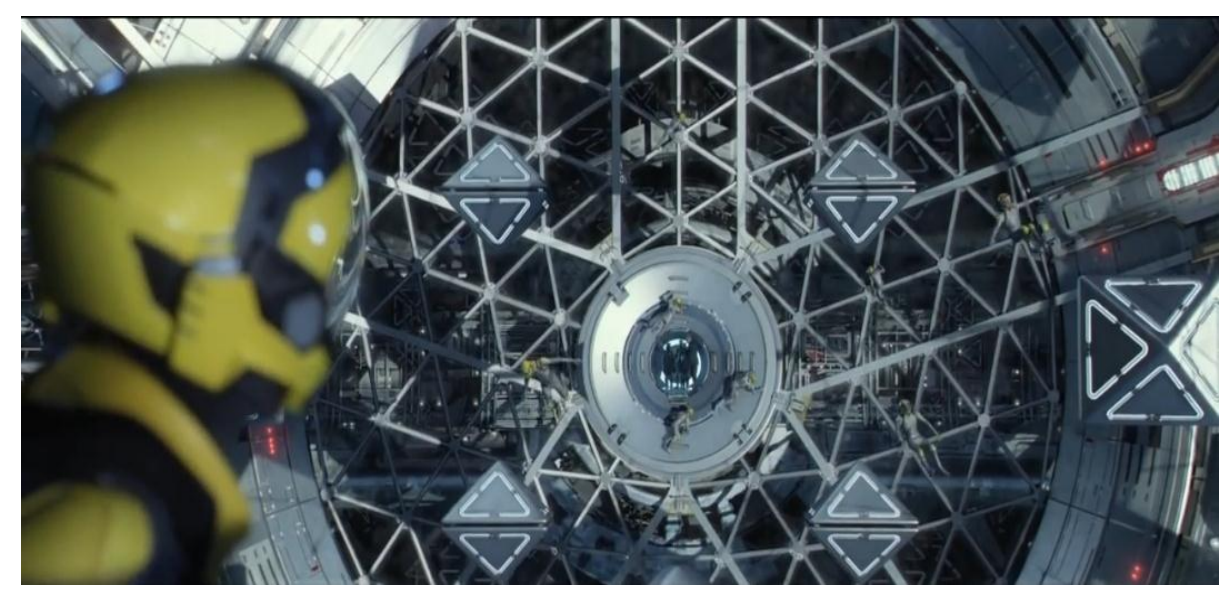

Dans cet exemple, la manipulation des décors s'adosse à la modernité des signifiants imagés que ce soit dans les motifs intérieurs des vaisseaux spatiaux ou extérieurs dans les simulations de guerre et de bombardements. Ces images à dominante métallique incitent le spectateur à s'immerger dans l'anecdote à l'aide d'une technologie cinématographique mise en scène au service de l'épopée militaire.

À cette représentation de l'espace s'associe un trope imaginaire classique dans le cinéma de science-fiction: celui de l'antigravité. C'est un terme générique désignant le contrôle de la zone gravitationnelle engendrée par le globe terrestre. Ce leitmotiv constitue une partie intégrante de la culture scientifique imaginaire qui ne manque pas à être exploitée dans la représentation visuelle du roman notamment dans les épisodes d'entraînement des combattants. Il importe alors de souligner l'empreinte du superviseur artistique du film à ce sujet. En effet, c'est ce façonneur qui manipule les décors où se déroule le récit 
filmique et aide à transposer à l'écran les technologies de l'espace à travers les représentations graphiques fictives qu'il élabore. Ceci dans le but d'incarner des contrées virtuelles intrafictionnelles ${ }^{(22)}$ décrites dans le roman dans une incarnation visuelle passionnante. 2. Les couleurs et les lumières:

Puisqu'une multitude de signes s'imbrique dans toute expérience audiovisuelle, nous ne pouvons passer outre le jeu des couleurs et du clair/obscur. En effet, la quasi-totalité des évènements du film sont des scènes d'intérieur où l'obscurité traduit l'ambiance rigide et tendue des combats. La plupart des images du film sont sombres avec une domination de la couleur noire, synonyme du danger.

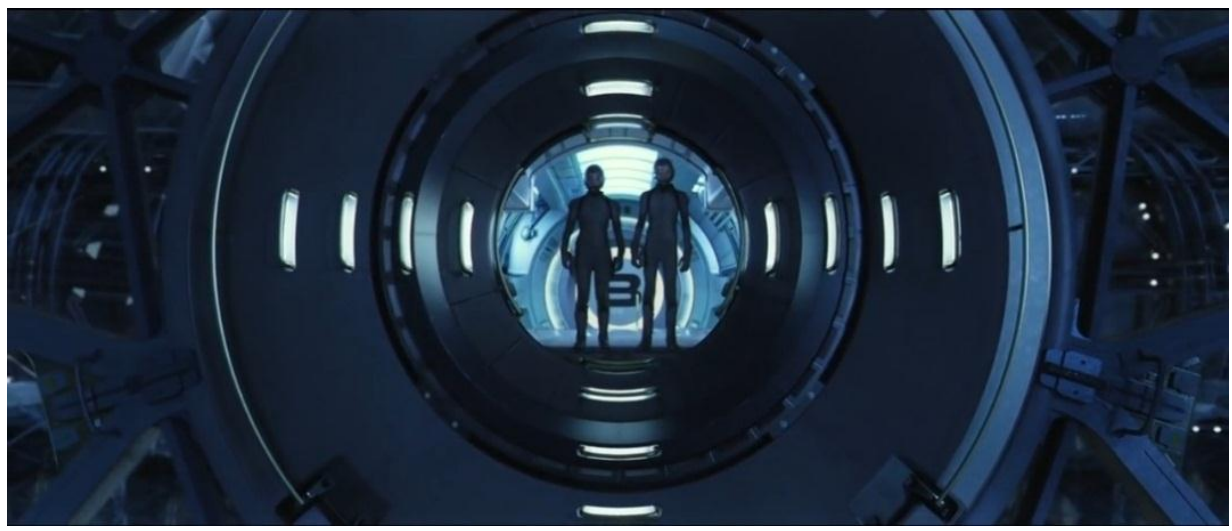

Les séquences des rêves et des hallucinations sont en sépia. Les dernières scènes en dehors de la station spatiale portent également des teintes tertiaires envahies par la couleur marron. En ce qui concerne le côté pictural, le traitement de la lumière est d'une extrême importance. Les simulations de guerre regorgent de

(La science-fiction du lisible au visible ...) Dr. Tarnime Mohamed Réda 
lumières laser dans les armes imaginaires comme les pistolets à rayons et les faisceaux de la mort. Ces derniers constituent une mine d'idées concernant l'usage du laser dans l'espace, variante science-fictionnelle par excellence.

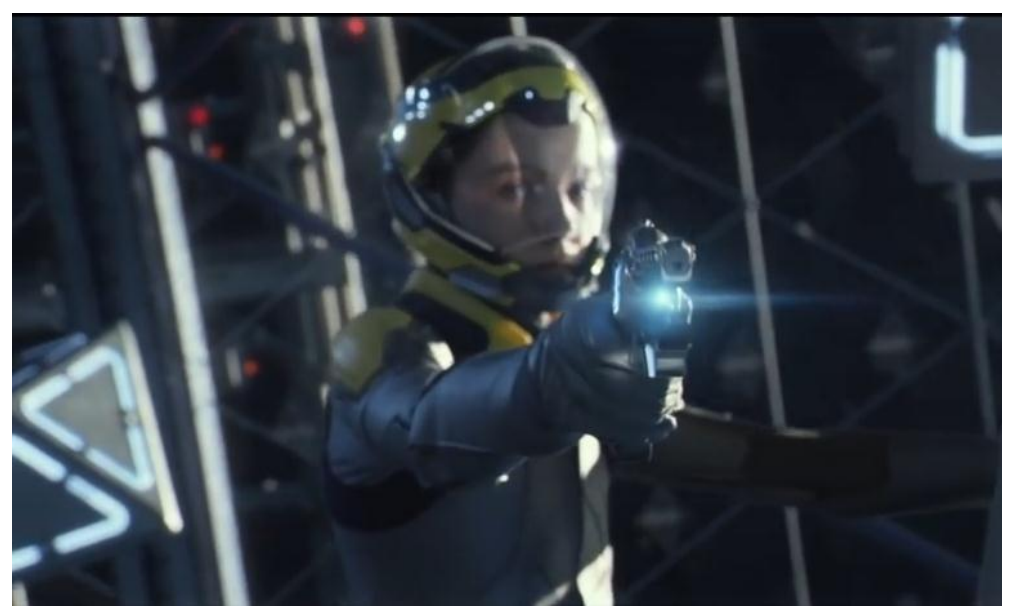

\section{Les cadres et l'échelle des plans:}

Le directeur de la photographie du film est l'australien Donald McAlpine. Si nous examinons son choix des cadres, nous apercevrons qu'ils sont remarquablement symboliques. Alors que, dans les scènes du début de la mission, l'accent était mis sur le Général Rakham le vainqueur de la première attaque contre les Doryphores, dans le reste des séquences, c'est Ender qui occupe toujours le devant de la scène. Cette interversion met en relief le renversement des forces dans cette situation où seuls les jeunes combattants pourront réaliser les objectifs et garantir la survie de la race humaine et soulignent également l'émergence du futur

(La science-fiction du lisible au visible ...) Dr. Tarnime Mohamed Réda 
héros chargé de rétablir le cours des événements. Les captures d'écrans suivantes constituent une preuve démonstrative.
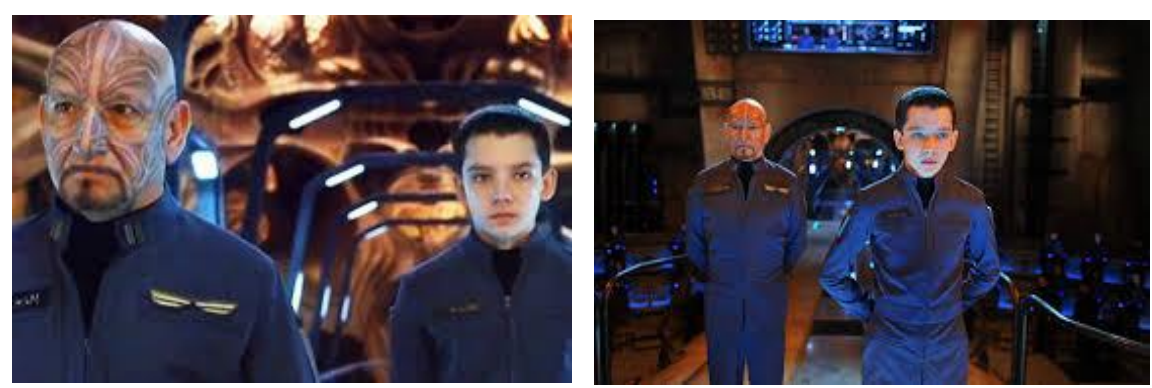

(23)

À cette idée de la particularité de la photographie se rejoint celle de la caméra subjective. Le plus souvent la découverte des décors se fait à travers le principe de «l'aeil enregistreur» ${ }^{(24)}$. Dans ce cas, la description cinématographie présentée à partir du point de vue d'un personnage précis se substitue aux pauses descriptives du roman en assumant la fonction d'un observateur humain. Le cadre ci-dessous présente un exemple de scène purement subjective qui adopte le point de vue du Colonel Graff en témoignant l'entraînement du jeune guerrier.

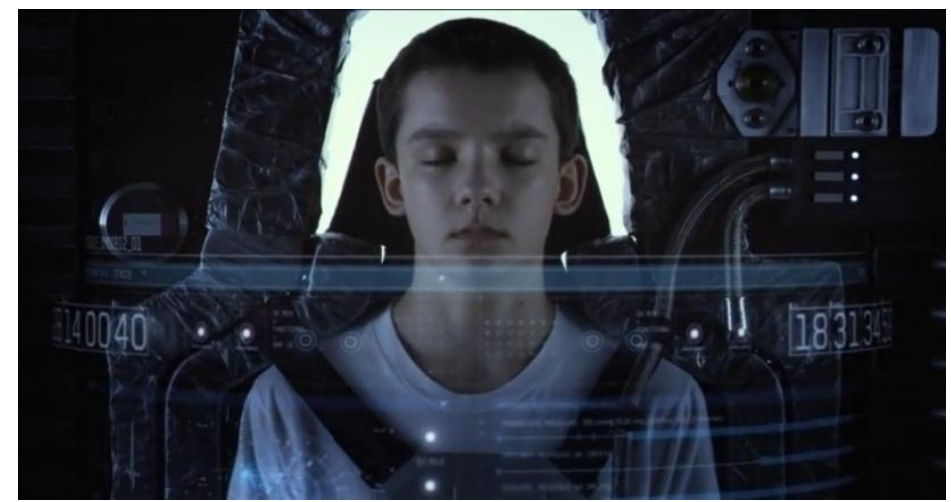

(25)

(La science-fiction du lisible au visible ...) Dr. Tarnime Mohamed Réda 1602 
À cet exemple de cadres s'associent ceux qui présentent un point de vue moins subjectif, dans lesquels la caméra se rejoint à l'un des personnages qui perçoit la scène et dont la présence demeure visible à l'écran. Il est vu par arrière et son regard se tourne vers le spectacle comme le montre l'exemple suivant:

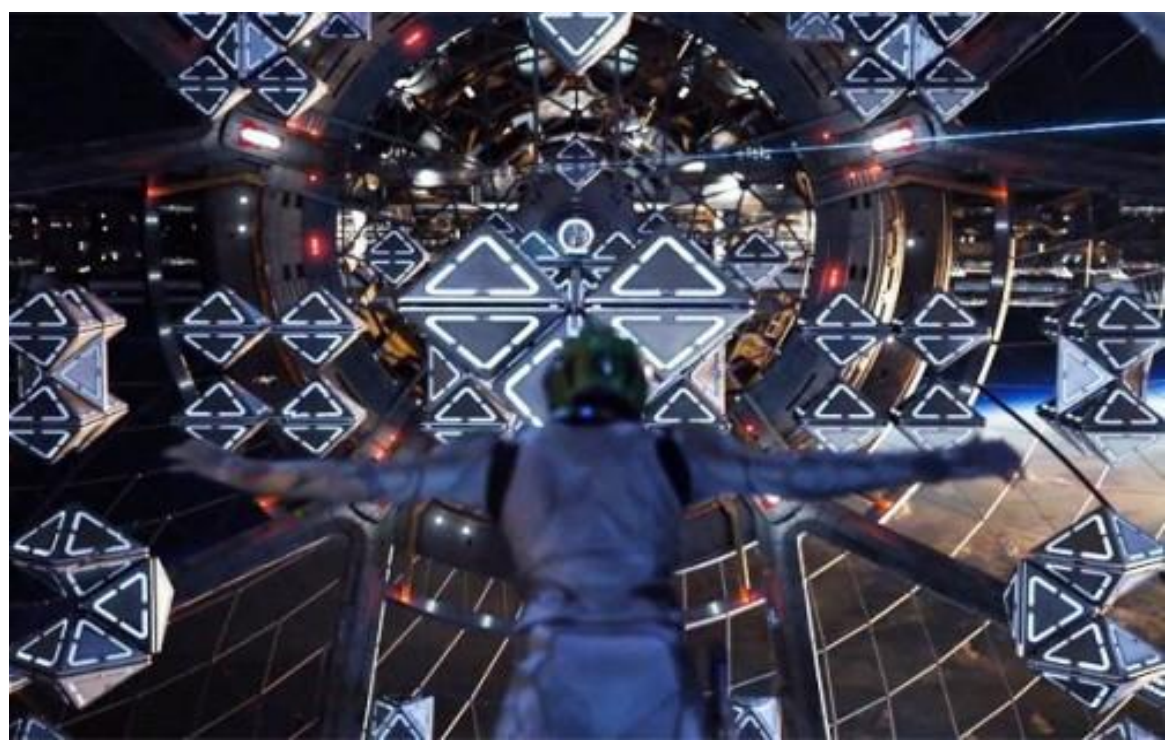

En addition, l'angle de prise de vue est chargé de significations. Nous distinguons principalement deux perspectives: la plongée et la contre plongée. Généralement, la plongée est particulièrement sollicitée dans les épisodes qui illustrent les simulations des combats. Elles donnent au public l'impression de dominer la scène et de se mêler aux événements. Quant à la contre-plongée, elle sert à rendre le personnage plus impressionnant et met surtout en relief le génie du jeune tacticien de guerre face à la grandeur du cosmos et à la gravité de l'ennemi qu'il affronte.

(La science-fiction du lisible au visible ...) Dr. Tarnime Mohamed Réda 


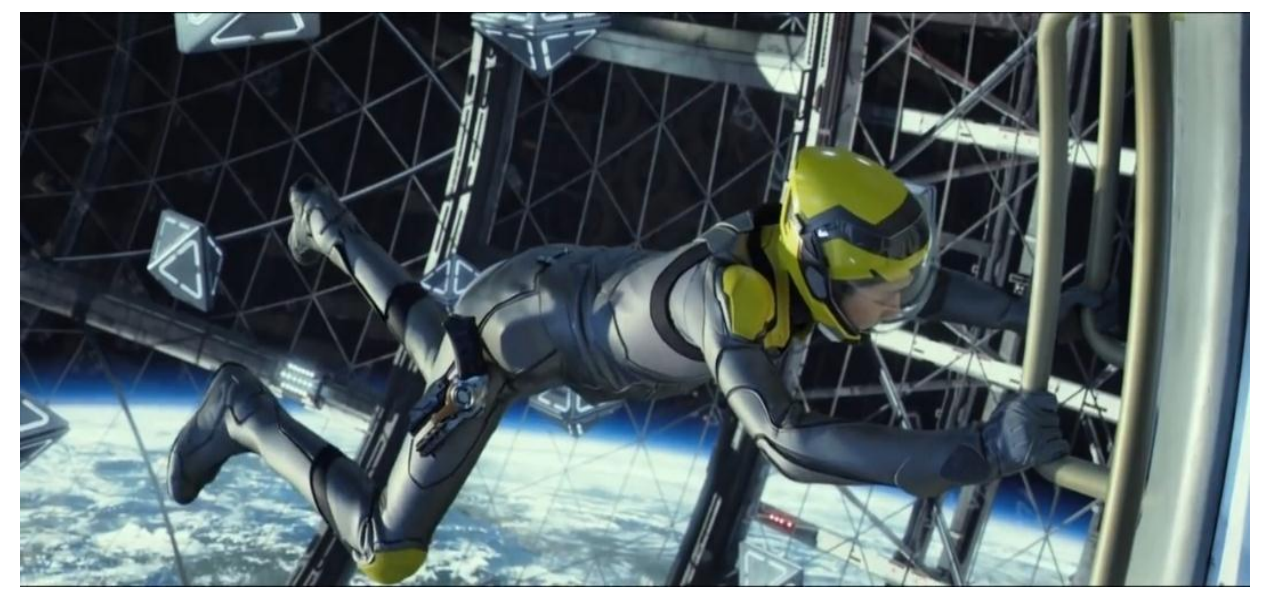

Considérons par exemple ce cadre photographié à l'aide d'une contreplongée dans lequel le héros franchit la salle de combat et apparaît en apesanteur comme s'il s'envolait dans le néant. L'angle de prise de vue donne cette impression due à la neutralisation de l'attraction dans le dispositif réalisé à des fins de vols spatiaux et de simulation numérique de guerres. Cet exemple des scènes qui ont lieu dans les vaisseaux interstellaires sert à introduire des effets de découverte et de surprise et permet également un renouvellement dans le matériau visuel à travers les interfaces élaborées. Ces séquences en apesanteur photographiées dans la zone entraînement sont vraiment ébouriffantes.

Aussi, les mouvements de la caméra contribuent-ils à créer un rythme accéléré notamment dans les scènes d'affrontement afin d'illustrer tour à tour des actions rapides et violentes tout en communiquant au lecteur la pression du temps. Ils servent également à créer des plans panoramiques mettant en évidence l'ampleur de l'espace interstellaire qui abrite les séquences de 
combats. Le montage du film soutient simultanément une logique esthétique de la discontinuité. Les coupes et la fragmentation alternée des plans utilisés par les monteurs Zach Staenberg et Lee Smith sont très significatifs à cet égard. Ils sont un facteur essentiel dans la production du discours filmique car ils montrent la lutte acharnée de la flotte des enfants-guerriers.

D'autres éléments supplémentaires jouent un rôle non moins important par leur capacité de « de conférer une consistance optique aux représentations mentales générées pendant la lecture » ${ }^{(26)}$ qu'il conviendrait d'analyser dans les volets suivants.

4. Les séquences techniques en dimension 3D:

Il demeure important de signaler l'apport des nouveaux outils informatiques capables de projeter le spectateur dans des univers artificiels simulateurs du réel. En addition à la vastitude des terrains de combats, l'usage de la nouvelle technologie fantastique s'impose également lorsqu'il s'agit de créer une représentation imaginaire de la race des ennemis. Le film nous présente une incarnation figurative des Doryphores comme étant des créatures dotées d'une intelligence singulière. 


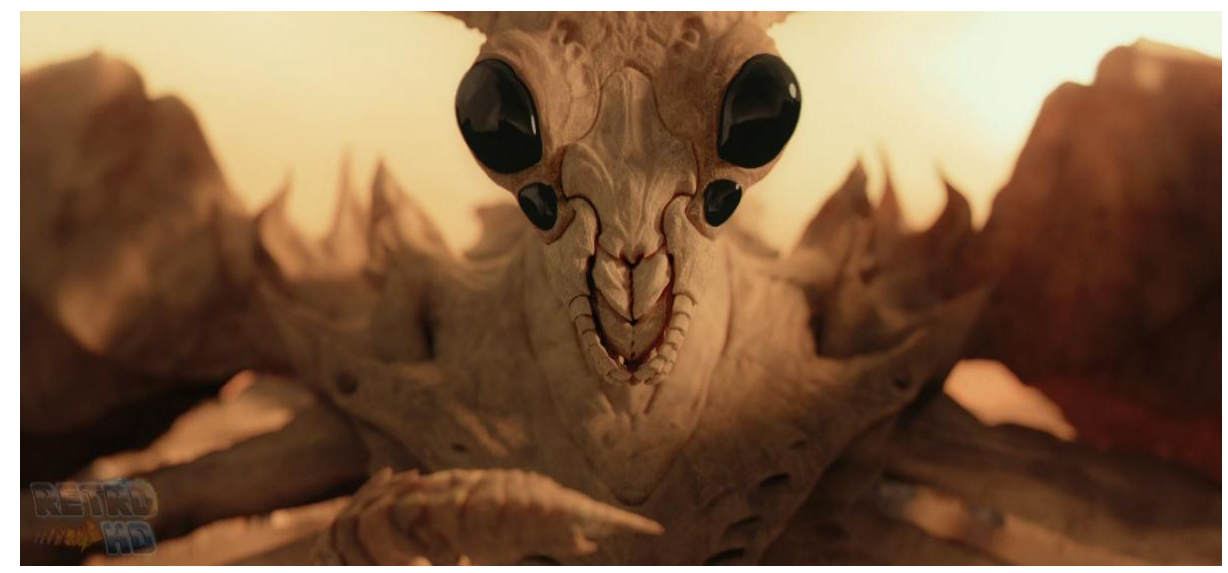

C'est ainsi que sont figurés les adversaires, imaginés à la base de critères animaliers. Dans la science-fiction, l'idée de l'affrontement interracial prime sur les conflits de guerre. Elle en constitue un véritable topo: celui de la rencontre avec l'extraterrestre. Ce dernier est surtout la métaphore de l'Autre; de l'inconnu qu'il faut combattre. Ses invariants structurels constituent l'un des piliers primordiaux des films d'aventures dans l'espace. Il est à souligner aussi que la confrontation entre les deux races ouvre un champ significatif de comparaison où chaque espèce mesure sa puissance et ses faiblesses. "Ecrire l'alien, c'est presque nécessairement écrire le conflit » ${ }^{(28)}$.

En outre, l'usage des technologies numériques et des effets spéciaux sert à donner des impressions de flou artistique et d'imprécision volontaire à l'image. Il ajoute des effets de profondeur inhérents à l'addition de la dimension 3D. Ceci s'exprime dans la finesse de la manipulation visuelle des

(La science-fiction du lisible au visible ...) Dr. Tarnime Mohamed Réda 
constructions mentales du héros dans ses rêves et ses hallucinations où «la fiction se virtualise à grands pas » ${ }^{(29)}$. Ces scènes aux teintes sépia revêtent un aspect émouvant grâce aux technologies sophistiquées mises en œuvre afin de concrétiser les idées contradictoires qui sillonnent l'esprit du protagoniste incarcéré dans ses illusions. L'exemple le plus significatif est le jeu numérique dans lequel Ender, insère inconsciemment la figure de sa sœur Valentine; épisode qui met en relief la forte pression psychique qui accable le héros.

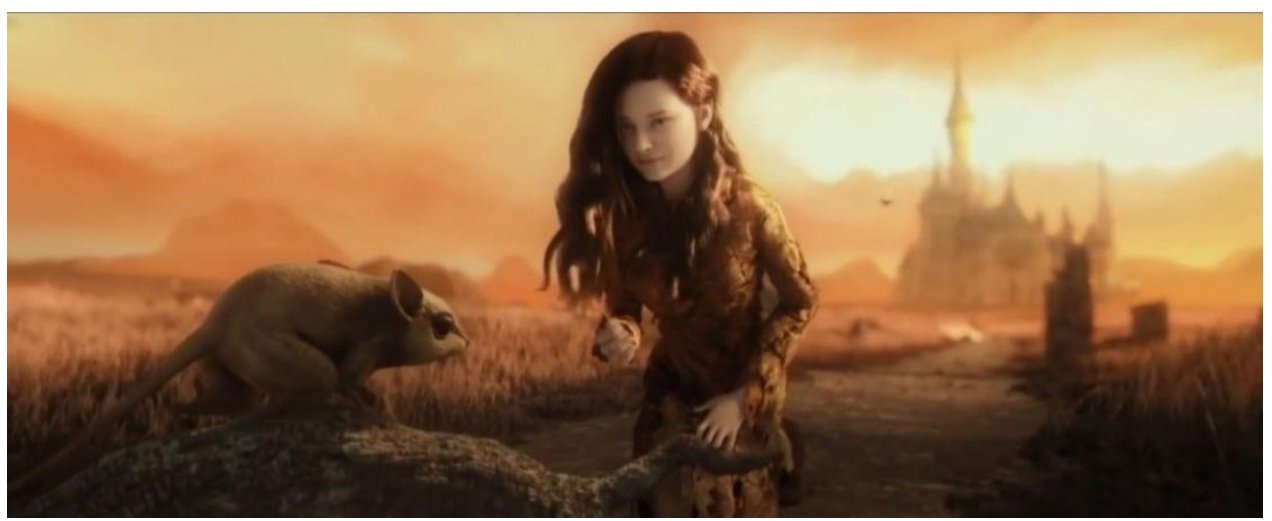

\section{Les costumes:}

Comme les acteurs prêtent aux personnages du roman leurs corps, leurs voix, et leurs personnalités, ils doivent se munir d'une allure attrayante portant des vêtements choisis par le réalisateur afin d'interpréter ces caractères. Le genre de la science-fiction, comme nous l'avons mentionné, est surtout le territoire de l'art visuel, il doit capter les regards et éblouir les spectateurs. C'est la raison pour laquelle le choix de la tenue constitue une composante primordiale de toute réalisation filmique imaginaire.

(La science-fiction du lisible au visible ...) Dr. Tarnime Mohamed Réda 


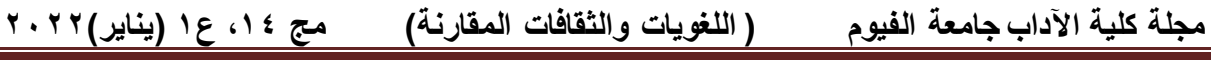
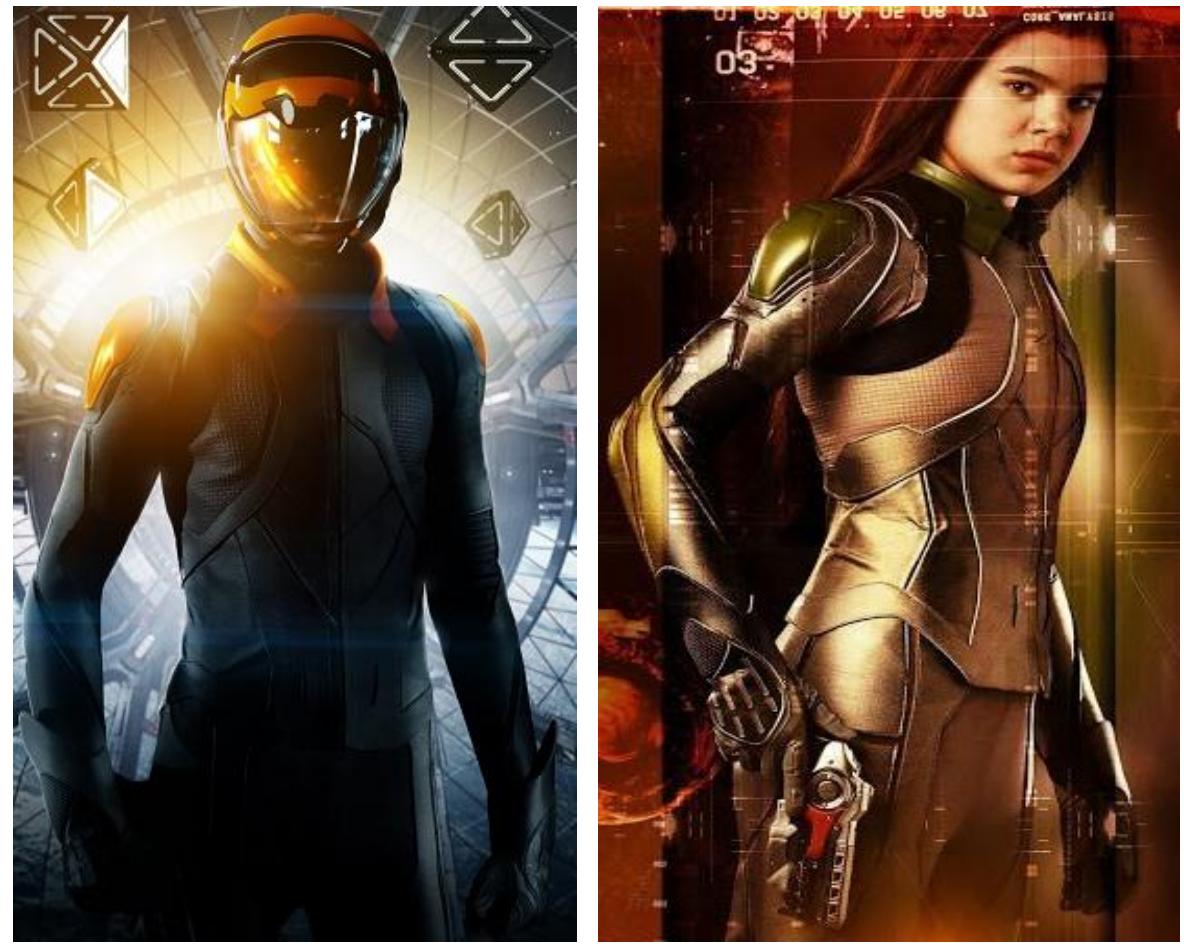

(30)

Dans La stratégie Ender, les costumes sont réalisés par Christine Bieselin Clark. Ils sont pour la majorité des habillements imaginaires éclatants d'allure plastique. Confectionnés à base métallique, ils transforment les combattants en de somptueuses créatures revêtues de matériau chatoyant, indissociable du contexte intersidéral. La création des costumes repose notamment sur les vestes des astronautes comme source d'inspiration, lesquelles sont fabriquées de manière à assurer un système de protection par le truchement de tissus vinyles qui maintiennent la sécurité des envoyés de l'espace. Cette splendeur de l'habillement des acteurs transporte le spectateur vers une ambiance d'« Ailleurs

(La science-fiction du lisible au visible ...) Dr. Tarnime Mohamed Réda 
» extraterrestre. Qu'ils soient de sexe masculin ou féminin, les jeunes guerriers revêtent le même costume verni. Ainsi, «les matières aux mille reflets si joliment mises en valeur ${ }^{(31)}$ flattent les regards de l'audience et exercent un attrait particulier sur les spectateurs. Ajoutons à cela que l'ordinateur pourrait parfois faire partie de la pièce vestimentaire des guerriers de l'espace. En Effet, Ender a été soumis à des expériences depuis sa tendre enfance. Au début du film, le relais implanté dans son cortex cérébral était présenté comme un équipement informatique interactif qui délivre des messages virtuels permettant d'observer ses réactions.

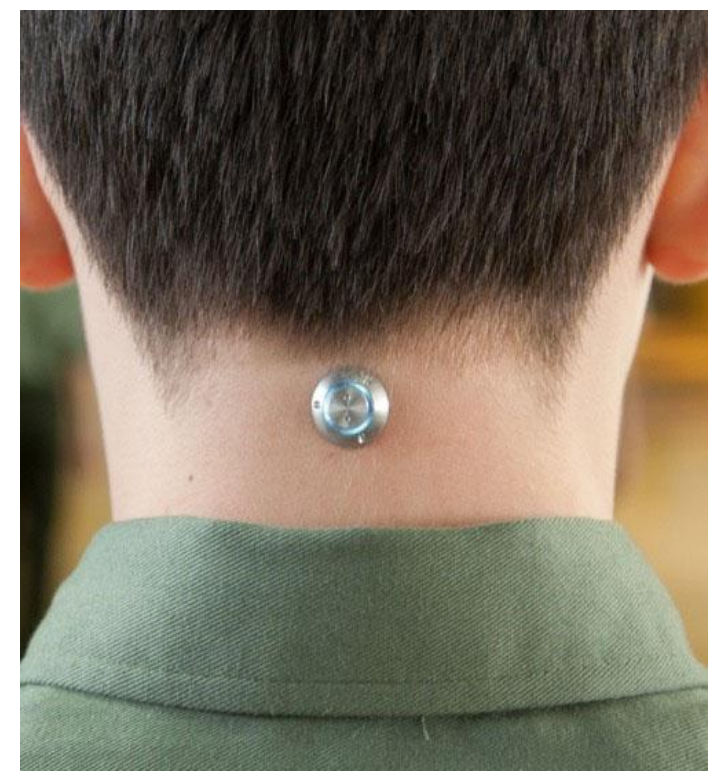

(32)

Par la suite, cet outil technique se révèle inextricable des vestes d'entraînement, où la tenue renferme un appareil de reconnaissance vocale associé à un microphone intégré qui

(La science-fiction du lisible au visible ...) Dr. Tarnime Mohamed Réda 
incarne la télésurveillance par les commandants. Ainsi, la sciencefiction est toujours considérée comme étant une machine d'idées fondée sur le développement des nouvelles technologies inédites.

Après avoir étudié le décor, les lumières, les jeux de la caméra, le choix des plans et des costumes, il s'avère donc que tous ces signifiants opèrent comme étant le vecteur principal du sens au septième art dans lequel l'image muette est à elle seule capable de suppléer l'essence de la finesse verbale. Or, d'autres éléments supplémentaires enrichissent les composants filmiques comme les effets sonores entre autres.

\section{La bande sonore:}

La musique pourrait transformer le film en une sorte de concert. Les effets musicaux constituent l'un des éléments cruciaux du langage cinématographique, ils assurent une relation de complémentarité entre les diverses composantes du récit filmique. Ces «effets d'enrichissement » (33) ont un impact particulier qui sert à émouvoir le public et à le garder toujours éveillé. Les supports sonores du film sont réalisés par le compositeur musical Steve Jablonsky. Dans quelques scènes, les sons créent un environnement émotionnel et aident à concevoir une atmosphère générale fondée sur la perception simultanée du bruitage et des images. Dans d'autres, ils peuvent parfois faire peur car le ton du film est très dur, surtout lorsqu'ils traduisent le suspense et le milieu tendu des simulations et des batailles. Par 
leurs différentes fonctions, ils aident à stimuler la concentration et soutiennent l'immersion du public dans l'ambiance générale du spectacle.

Aussi, la bande sonore englobe -t- elle la voix-off qui apporte des précisions et ajoute quelques informations supplémentaires. Soulignons que le héros était voué à un isolement quasi-permanent tout au long de sa formation militaire. Les détails concernant sa tactique de pensée et sa psychologie dans le roman sont substitués par des récitatifs qui se superposent aux dialogues et servent à ajouter des commentaires aux épisodes. Le son comprend également tous les bruits relatifs à l'usage des armes à rayons, aux déplacements des guerriers, à la prise de position des combattants et même au rythme accéléré de la respiration qui accompagne l'apogée de la tension dramatique.

Ainsi, l'importance de la musique pourrait se mesurer aux lacunes produites par son absence. Nous constatons donc que son rôle ne se limite pas à une simple toile de fond. La bande sonore représente l'une des clés importantes de l'art filmique où s'allient l'audio et le visuel dans une fusion des signifiants cinématographiques afin de créer un véritable spectacle acoustique.

\section{Le rythme et les détails accentués:}

L'étude de la composition chronologique constitue un dernier point important que nous souhaiterons élucider. Comme 
déjà signalé, la discordance entre le facteur temporel dans le roman et le film pose un grand défi au cinéaste. La rythmique du septième art a bien évolué, elle ne cesse de s'accélérer surtout lorsqu'il s'agit d'un film de fiction scientifique. L'adaptation doit stimuler le spectateur et l'accrocher en lui assurant une dose maximum de vivacité tout en évitant la monotonie et les digressions et surtout en variant le tempo narratif.

Pour ce faire, il est indispensable de varier les scènes d'actions intenses et les scènes de pauses. En effet, Gavin Hood a étoffé la montée en puissance du jeune Wiggin en parallèle avec l'aggravation de la tension relative au processus d'encadrement militaire. Cette alternance entre les deux types d'épisodes précités souligne les sentiments du héros écartelé entre son objectif ultime de victoire et les angoisses et les questionnements qui envahissent son esprit enfantin. Ainsi, le temps du film est voué à être condensé car il demeure toujours soumis à la technique de vitesse qui en fait une version abrégée de l'œuvre littéraire.

En outre, une variante étroitement liée au rythme est celle du flashback. Elle consiste à faire une déconstruction des événements et à y insérer des arrêts permettant de présenter des détails indispensables à la compréhension. Ces retours en arrière sont intégrés pour combler une lacune dans le récit, ou fournir un renseignement important concernant l'un des personnages. Dans le film, ils insèrent des éléments relatifs au dynamisme de la 
mémoire du héros et révèlent des détails concernant sa relation avec sa famille et notamment sa sœur. Il est à noter que les flashbacks dans le film sont dans leur totalité déclenchés à partir de la technique cinématographique du fondu enchaîné qui consiste à superposer un plan présent à un plan du passé tout en introduisant un changement dans les composantes visuelles comme la variation des couleurs en teintes sépia.

D'emblée, l'ouverture et la clôture du récit filmique sont des moments stratégiquement très significatifs. Si le film s'avère complètement fidèle au roman dans son début, il détourne la fin et opte vers une fin plus ouverte et surtout indécidable en présentant «une cause suspendue » ${ }^{(34)}$. La dernière simulation s'est révélée la plus difficile et le seul moyen de marquer la victoire semblait de sacrifier la vie d'un millier de combattants en abandonnant la flotte entière afin de détruire la planète qui abrite les reines de l'ennemi. Ce dénouement met l'accent sur les séquelles psychiques de la vie dans une école de guerre dans laquelle les jeunes enfants sont amenés à affronter les risques et transformés en de personnes impitoyables, comme de véritables « machines à tuer ».

Une fois terminé sa mission et après avoir réussi, Ender découvre que ce n'était pas une simulation mais une véritable bataille qui le transforme en un assassin responsable du xénocide de toute une espèce. Il comprend ainsi que tout ce drame était dû à 
un manque de communication puisque les Doryphores n'ont jamais essayé d'attaquer les terriens pendant une cinquantaine d'années. Les reines ont freiné la colonisation car elles contrôlent les ouvrières par télépathie et depuis, ce sont les humains qui les contre-attaquent craignant une nouvelle offensive. Après ce twist final, le héros découvre une larve de Doryphore qu'il tente de protéger pour remédier à son sentiment de culpabilité. Par conséquent, il décide de ne plus revenir sur terre et se lance à la recherche d'une planète habitable qui pourrait l'abriter. Cette finale accomplit l'arc transformationnel du héros mais laisse simultanément la porte ouverte à toute investigation supplémentaire. Elle répond à une tendance cinématographique moderne qui présente un large champ d'interprétation et appelle à une continuation du récit que chaque spectateur est appelé à combler à son gré.

C'est ainsi que le film varie le rythme de la narration en mettant en relief quelques détails et en écartant d'autres éléments non essentiels qui sont jugés accessoires.

«La force du cinéma [...] est dans cette polysémie qui peut espérer rejoindre et, parfois même, dépasser les capacités expressives de la langue et de la littérature » ${ }^{(35)}$. 
En conclusion, l'objectif de notre recherche est de démontrer que des liens solides associent l'art littéraire à l'art filmique. D'une part, le septième art a constamment puisé dans la littérature pour se nourrir. D'autre part, si l'œuvre possède une densité notable, elle s'enrichit surtout à être réinterprétée et transposée à l'écran. Dans le cadre d'une approche comparative, nous avons effectué un mouvement alternatif entre le verbal et le visuel. À travers une analyse des repères entre les deux types de narrations, nous avons éclairé la richesse latente de la manipulation cinématographique et surtout des procédés utilisés par le «génie créateur».

Dans un premier temps, nous avons souligné quelques données propres au romancier et au réalisateur afin de rendre compte de la particularité de chaque entreprise du point de vue de son acte producteur. Dans un second temps, nous avons mis en relief que les systèmes narratifs romanesque ou filmique s'érigent autour des relations entre des personnages en évolution constante dans le temps et dans l'espace. Dans un troisième temps, nous avons décortiqué l'étape transitoire qui constitue la phase intermédiaire chargée de transformer le récit littéraire en un spectacle cinématographique.

Après avoir étudié les spécificités de chacune des deux structures artistiques, nous avons jugé important de fonder notre étude sur l'exemple de l'adaptation du roman La stratégie Ender.

(La science-fiction du lisible au visible ...) Dr. Tarnime Mohamed Réda 
Même si, dans le cadre restreint de cet article, il était difficile d'élaborer une analyse détaillée du film, nous avons toutefois tenté d'étudier les techniques de mise en scène les plus significatives se rapportant au domaine science-fictionnel. Nous avons donc constaté que le cinéma impose une profusion de détails particuliers par le truchement de maints procédés de réalisation: casting, décor, lumière, cadrage, échelle des plans, angle de prise de vue, procédés multidimensionnels, costumes, musique, rythme. Nous avons enfin conclu que le cinéma et la littérature sont deux arts complémentaires quoique différents. L'œuvre adaptée est un produit spécialisé dans lequel les éléments livresques s'effacent au profit d'une abondance de signifiants plus denses sur le plan narratif et émotionnel. Aussi, la mise en scène est -t- elle un acte de création qui vise à la production du sens, tout en employant une proportion bien dosée des différentes techniques iconiques et auditives. Ce processus de scénarisation repose essentiellement sur l'homogénéité des signifiants filmiques visuels, sonores et interprétatifs.

En définitive, le transfert du «prestige du livre » à l'écran est une entreprise de grande valeur qui déborde le dilemme fidélité/infidélité. L'exploration de ce territoire mérite d'être décortiquée tout en prenant en considération l'impossibilité d'une parfaite équivalence entre littérature et cinéma; thématique qui 


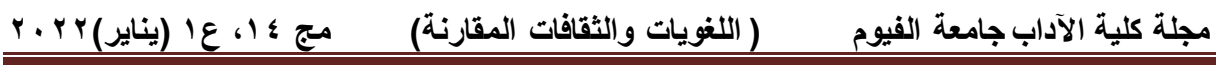

demeure d'actualité et stimule la curiosité des chercheurs au cours des dernières années.

(La science-fiction du lisible au visible ...) Dr. Tarnime Mohamed Réda 


\section{Notes:}

(1) ASTRUC, Alexandre, Du stylo à la caméra... Et de la caméra au stylo, 1992, p. 327. Le concept de la caméra stylo a été présenté pour la première fois dans l'article «Naissance d'une nouvelle avant-garde » paru en mars 1948.

(2) Ender's game (La Stratégie Ender) est un film américain de science-fiction réalisé par Gavin HOOD en 2013. La version française est disponible via le lien:

https://www.youtube.com/watch?v=PHF7vTl2byw. Pour la traduction française du roman, nous nous référons à Orson Scott CARD, $L a$ Stratégie Ender, OPTA, coll. Club du livre d'anticipation, 1986, traduit de l'anglais par Daniel LEMOINE, 460 pages. L'œuvre originale a été publiée en 1985 sous le titre de Ender's game à New York. Elle a remporté la même année les prix Nebula et Hugo (léquivalent des Oscars en littérature SF et fantastique) et fait figure de classique chez les amateurs du genre. $C f$., CESBRON, Mathilde, «La Stratégie Ender et Harrison Ford boycottés par la communauté gay ». URL:

https://www.lefigaro.fr/cinema/2013/07/10/03002-

20130710ARTFIG00365--la-strategie-ender-et-harrison-ford-

boycottes-par-la-communaute-gay.php, consulté le 6 avril 2021.

(3) BEGUIN-VERBRUGGE, Annette, Images en texte, images $d u$ texte: dispositifs graphiques et communication écrite, Villeneuve d'Ascq, Presses universitaires du Septentrion, coll. Informationcommunication, 2006, p. 203.

(4) PELLETIER, Esther, « Texte littéraire et adaptation cinématographique : la rencontre de deux systèmes », numéro 12, 1984. URL: $\quad$ http://collections.cinematheque.qc.ca/articles/textelitteraire-et-adaptation-cinematographique-la-rencontre-de-deuxsystemes/, consulté le 4 avril 2021.

(5) MORENCY, Alain, "L'adaptation de la littérature au cinéma », In Horizons philosophiques, volume 1, numéro 2, 1991, p. 104. DOI: https://doi.org/10.7202/800874ar, consulté le 29 mars 2021.

(6) $\mathrm{Ibid}$, p. 109.

(La science-fiction du lisible au visible ...) Dr. Tarnime Mohamed Réda 
(7) LAROCHE, Daniel, "Du livre au film », Revue Le Carnet et les instants (dossier Littérature \& Cinéma), $\mathrm{n}^{\circ}$ 185, mars 2015. URL: https://www.revues.be/le-carnet-et-les-instants/80-le-carnet-et-lesinstants-185/144-du-livre-au-film-dossier-litterature-cinema, consulté le 30 mars 2021.

(8) MERAD, Soumeyya, "Littérature et cinéma L'adaptation une autre figure de l'intertextualité », Revue des sciences humaines, numéro 48, 2017, p. 185.

(9) PELLETIER, Esther, art. cit, p. 38.

(10) MERAD, Soumeyya, art. cit., p. 183.

(11) Ibid., p. 185.

(12) LAROCHE, Daniel, art. cit.

(13) LABRECQUE, Maxim, "L'adaptation cinématographique : regard sur une pratique polémique », Séquences : La revue de cinéma, numéro 302, 2016, p. 54.

(14) PELLETIER, Esther, art. cit, p. 41.

(15) LAROCHE, Daniel, art. cit.

(16) FAWZI, Mohdeb, «Adaptation cinématographique et production littéraire », URL:

https://www.academia.edu/32846485/Chapitre_II_Adaptation_cin\%C3

\%A9matographique_etproduction_litt\%C3\%A9raire, consulté le 4 avril 2021.

(17) Nous empruntons la photo au site

https://www.premiere.fr/Series/La-Strategie-Ender-le-blockbuster-

adapte-en-serie, consulté le 4 avril 2021. En ce qui concerne les photos sans références, nous les avons enregistrées en captures d'écran du film afin de les employer pour appuyer nos analyses.

(18) LAROCHE, Daniel, art. cit.

(19) Photos empruntées au site

https:/www.pinterest.com/pin/376121006353195882/,

https://www.pinterest.com/endernet/colonel-hyrum-graff/ , consulté le 6 mai 2021.

(20) LAROCHE, Daniel, art. cit.

(La science-fiction du lisible au visible ...) Dr. Tarnime Mohamed Réda 
(21) WOODS, Arthur, «L'exploration de l'espace à travers les artistes et les écrivains », In Collectif, Les nouvelles technologies dans la science-fiction appliquées au domaine spatial, Textes réunis par $\mathrm{P}$. Gyger, D. Raitt et A. Woods, In European Space Agency, numéro BR176, URL: https:/core.ac.uk/download/pdf/25366779.pdf, 2002, p. 9.

(22) Cette expression désigne "les espaces virtuels décrits dans les romans et les films de science-fiction. Il s'agit en quelque sorte d'espaces virtuels au carré, des fictions dans la fiction, enchâssés comme des poupées russes dans des univers fictionnels, littéraires ou cinématographiques ». THUILLIER, Guy, «Entre fiction, simulacre et réalité : les avatars de l'espace virtuel », Carnets de géographes, numéro 2, Rubrique Carnets de recherches, 2011. DOI: https://doi.org/10.4000/cdg.2674, consulté le 15 avril 2021.

(23) Les photos sont empruntées au site

https://enderverse.fandom.com/wiki/Andrew_Wiggin, consulté le 6 mai 2021.

(24) MORRISSETTE, Bruce, « Problèmes du roman cinématographique », In Cahiers de l'Association internationale des études françaises, numéro 20, 1967, p. 279. DOI : https://doi.org/10.3406/caief.1968.914, consulté le 23 avril 2021.

(25) La photo est empruntée au site https://enderverse.fandom.com/wiki/Stasis, consulté le 6 mai 2021. (26) ANDA-MARIA, I., L'adaptation cinématographique des œuvres littéraires. Apories. Préjugés. Nouvelles perspectives, Thèse de doctorat Université BABES-BOLYAI, 2016, p. 7.

(27) La photo est empruntée au site http //sebiwandanslesetoiles.overblog.com/2014/04/la-strategie-ender.html, consulté le 6 mai 2021.

(28) LANGLET, Irène, «Guerres du futur, conflits du présent », In Imaginaires de guerre et autres conflits, numéro 5, 2015. DOI : https://doi.org/10.4000/carnets.311, consulté le 23 avril 2021.

(29) THUILLIER, Guy, art. cit.

(30) Photos empruntés au site

https:/www.pinterest.com/pin/320951910915866236/, consulté le 6 mai 2021.

(La science-fiction du lisible au visible ...) Dr. Tarnime Mohamed Réda 
(31) BILLY BOY, "L'attrait des combinaisons vinyles de l'espace », In Collectif, Les nouvelles technologies dans la science-fiction appliquées au domaine spatial, Textes réunis par P. Gyger, D. Raitt et A. Woods, In European Space Agency, numéro BR-176, URL: https://core.ac.uk/download/pdf/25366779.pdf, p. 6.

(32) Photo empruntée au site

https:/www.giantfreakinrobot.com/scifi/andrew-wiggin-enders-

game.html, consulté le 6 mai 2021.

(33) SALLES, Daniel, "L'adaptation cinématographique d'œuvres littéraires », In l'École des Lettres des collèges, Académie de Grenoble, 2005. URL: http://www.acgrenoble.fr/audiovisuel/articles.php?lng=fr\&pg=57, consulté le 27 avril 2021.

(34) Cf., AUMONT, Jacques, L'interprétation des films, A. Colin, 2017, p. 187.

(35) MORENCY, Alain, art. cit., p. 104.

(La science-fiction du lisible au visible ...) Dr. Tarnime Mohamed Réda 


\section{Bibliographie}

Nous rappelons que, sauf indication contraire, la ville d'édition dans notre étude est Paris.

\section{Corpus :}

Film: Gavin HOOD, Ender's game, 2013. La version française intitulee La Stratégie Ender est disponible via le lien: https://www. youtube.com/watch?v=PHF7vTl2byw.

Roman: CARD, Orson scott, La stratégie Ender, OPTA, coll. Club du livre d'anticipation, 1986, traduit de l'anglais par Daniel LEMOINE, 460 pages. (Euvre originale Ender's game, New York, 1985).

\section{Références :}

1) ANDA-MARIA, I., L'adaptation cinématographique des cuvres littéraires. Apories. Préjugés. Nouvelles perspectives, Thèse de doctorat Université BABES-BOLYAI, 2016.

2) Astruc, Alexandre, Du stylo à la caméra... Et de la caméra au stylo, 1992.

3) AUMONT, Jacques, L'interprétation des films, A. Colin, 2017.

4) BEGUIN-VERBRUGGE, Annette, Images en texte, images du texte: dispositifs graphiques et communication écrite, Villeneuve d'Ascq, Presses universitaires du Septentrion, coll. Informationcommunication, 2006.

5) BILLY BOY, "L'attrait des combinaisons vinyles de l'espace », In Collectif, Les nouvelles technologies dans la science-fiction appliquées au domaine spatial, Textes réunis par P. Gyger, D. Raitt et A. Woods, In European Space Agency, numéro BR-176, URL: https://core.ac.uk/download/pdf/25366779.pdf, consulté le 5 mai 2021. 6) BRUCE, Morrissette, «Problèmes du roman cinématographique », In: Cahiers de l'Association internationale des études françaises, $\mathrm{n}^{\circ}$ 20, 1968, p.p. 275-289. DOI: https://doi.org/10.3406/caief.1968.914 
7) CESBRON, Mathilde, «La Stratégie Ender et Harrison Ford boycottés parla communauté gay ». URL: https://www.lefigaro.fr/cinema/2013/07/10/03002-

20130710ARTFIG00365--la-strategie-ender-et-harrison-fordboycottes-par-la-communaute-gay.php, consulté le 6 avril 2021.

8) FAWZI, Mohdeb, «Adaptation cinématographique et production littéraire

https://www.academia.edu/32846485/Chapitre_II_Adaptation_cin\%C3

\%A9matographique etproduction litt\%C3\%A9raire, consulté le 4 avril 2021.

9) LABRECQUE, Maxime, "L'adaptation cinématographique : regard sur une pratique polémique », In: Séquences : la revue de cinéma, $\quad \mathrm{n}^{\circ} \quad 302, \quad 2016 . \quad$ p.p. 52-56. URL: https://id.erudit.org/iderudit/82186ac, consulté le 5 mai 2021.

10) LANGLET, Irène, "Guerres du futur, conflits du présent », In Imaginaires de guerre et autres conflits, numéro 5, 2015. DOI: https://doi.org/10.4000/carnets.311, consulté le 23 avril 2021.

11) LAROCHE, Daniel, Du livre au film (dossier Littérature \& Cinéma),URL: https://revues.be/le-carnet-et-les-instants/80-lecarnet-et-les-instants-185/144-du- livre-au-film-dossier-litteraturecinema, consulté le 5 mai 2021.

12) MERAD, Soumeya, " L'adaptation une autre figure de l'intertextualité », In: Revue des sciences humaines, $\mathrm{n}^{\circ}$ 48, Vol B, décembre 2017, p.p. 571-581.

13) MORENCY, A., "L'adaptation de la littérature au cinéma. Horizons philosophiques », 1 (2), 1991, p.p. 103-123. DOI: https://doi.org/10.7202/800874ar, consulté le 5 mai 2021.

14) MORRISSETTE, Bruce, « Problèmes $d u$ roman cinématographique », In Cahiers de l'Association internationale des études françaises, numéro 20, 1967. DOI : https://doi.org/10.3406/caief.1968.914, consulté le 23 avril 2021.

15) PELLETIER, Esther, " Texte littéraire et adaptation cinématographique: la rencontre de deux systèmes », In: Le cinéma : théorie et discours, $\mathrm{n}^{\circ}$ 12, décembre 1984. URL: 
http://collections.cinematheque.qc.ca/articles/texte-litteraire-etadaptation- cinematographique-la-rencontre-de-deux-systemes/, consulté le 5 mai 2021.

16) SALLES, Daniel, "L'adaptation cinématographique d'œuvres littéraires », In L'École des Lettres des collèges, Académie de Grenoble, 2009. URL: http://www.acgrenoble.fr/audiovisuel/articles.php? $\operatorname{lng}=$ fr\&pg=57, consulté le 27 avril 2021.

17) THUILLIER, Guy, "Entre fiction, simulacre et réalité : les avatars de l'espace virtuel », Carnets de géographes, numéro 2, Rubrique Carnets de recherches, 2011. DOI: https://doi.org/10.4000/cdg.2674, consulté le 15 avril 2021.

18) WOODS, Arthur, «L'exploration de l'espace à travers les artistes et les écrivains », In Collectif, Les nouvelles technologies dans la science-fiction appliquées au domaine spatial, Textes réunis par $P$. Gyger, D. Raitt et A. Woods, In European Space Agency, numéro BR176, URL: https://core.ac.uk/download/pdf/25366779.pdf, 2002.

\section{Sitographie:}

https://www.premiere.fr/

https://www.pinterest.com/

https://enderverse.fandom.com/

http://sebiwandanslesetoiles.over-blog.com/

https://www.giantfreakinrobot.com

(La science-fiction du lisible au visible ...) Dr. Tarnime Mohamed Réda 
الخيال العلمي بين المقروء والمرئي

\section{ملخص}

يهدف هذا البحث إلى إثبات الروابط الوثيقة بين الفن الروائي والفن السينمائي. لطالما اتجه الفن السابع لاقتباس موضوعاته من التراث الأدبي ومن ناحية أخرى فإن العمل الأدبي يكتسب بعدا اخر عند تجسيده من خلال عمل فني مرئي. فمن خلال دراسة مقارنة، تقوم الباحثة بعمل تحليل للهيكل الفني الخاص بالبناء الروائي من جهة والبناء السنمائي من جهة أخرى وذلك بهدف نوضيح الخصائص البنائية للهيكل الأدبي والدرامي كلا على حدى. وقد لجأت الباحثة لتطبيق هذا المنهج المقارن على الفيلم الأمريكي "العملية إندر" للمخرج العالمي جافين هود، وهو فيلم خيال علمي مقتبس من ون رواية أمريكية تحمل نفس العنوان. وقد قامت الباحثة بدراسة تحليلة لتفاصيل تحويل هذه الرواية إلي فيلم سينمائي من خلال مجموعة من التقنيات في الإخراج مثل تحليل اختيار الفنانين لتجسيد الثخصيات، الخلفيات والديكور، الإضاءة، التصوير وزوايا الرؤية، استخدام التقنيات ثلاثية الأبعاد، اختيار الأزياء والموسيقى، إيقاع السرد. فمن خلال تحليل هذه النقنيات تمكنت الباحثة من إلقاء الضوء على الثراء الذي تضيفه السينما إلى العمل الأدبي من خلال تحويله من النص المقروء إلى بناء سينمائي متعدد العناصر

الكلمات المفتاحية: سينما الخيال العلمي، الإخراج السينمائي، الأدب والسينما. 